\title{
Modelling Vascularity in Breast Cancer and Surrounding Stroma using Diffusion MRI and Intravoxel Incoherent Motion
}

\author{
Colleen Bailey ${ }^{1}$, Sarah Vinnicombe ${ }^{2}$, Eleftheria Panagiotaki ${ }^{1}$, Shelley A \\ Waugh $^{2}$, John H Hipwell ${ }^{1}$, Daniel C Alexander ${ }^{1}$, Kathryn Kitching ${ }^{2}$, Patsy \\ Whelehan $^{2}$, Sarah E Pinder ${ }^{3}$, Andrew Evans ${ }^{2}$, and David J Hawkes ${ }^{1}$ \\ 1 Centre for Medical Image Computing, University College London, Engineering \\ Front Building, London, UK, WC1E 6BT \\ colleen.bailey@ucl.ac.uk \\ 2 Dundee Cancer Centre, Ninewells Hospital and Medical School, Dundee, UK, DD1 \\ 9SY \\ 3 Breast Research Pathology, Research Oncology, Division of Cancer Studies, King's \\ College London, Guy's Hospital, London, UK, SE1 9RT
}

\begin{abstract}
Contrast-enhanced magnetic resonance imaging (MRI) has shown variation in the stroma with distance from the tumor and this correlates with histological microvessel density. To date, however, conventional diffusion MRI has demonstrated limited sensitivity to these changes. This study modelled the diffusion signal by intravoxel incoherent motion (IVIM) to obtain parameters related to the vasculature and tissue diffusion. This revealed a small vascular contribution to the signal in tumor and peri-tumoral stroma within $8 \mathrm{~mm}$. Monoexponential fitting performed worse than the IVIM model in tumor and stroma within $8 \mathrm{~mm}$, but was sufficient in more distal stromal regions where lower microvessel density is expected. Modelling diffusion MRI by IVIM provided a measure of vascularity that may complement information from DCE-MRI and yielded additional information about diffusion in the extravascular tissue.
\end{abstract}

Keywords: diffusion MRI, vascularity, stroma, modelling, IVIM, breast cancer

\section{Introduction}

As breast cancer treatment options increase, tools to predict tumor invasiveness and recurrence are needed. Changes in peri-tumoral stroma are potential markers of outcome. For example, signal enhancement from Dynamic ContrastEnhanced Magnetic Resonance Imaging (DCE-MRI) correlates with tumor recurrence [1] and survival [2]. There is also spatial variation: signal enhancement ratio becomes less prominent with distance from the tumor, an effect that correlates with microvessel density [3]. However, DCE-MRI signal at low temporal resolution depends on many physiological parameters, including the microvessel 
density, blood flow, vessel permeability and the extracellular space into which contrast agent leaks.

Diffusion MRI is also sensitive to the vasculature. The intravoxel incoherent motion (IVIM) model separates vascular pseudodiffusion from diffusion in the extravascular tissue and has recently been used to model the diffusion signal from breast tumors $[4,5]$, demonstrating a pseudodiffusive contribution to the signal of $6-10 \%$ for malignant tissues. This component was less than $2 \%$ in noncancerous fibroglandular tissue (FGT), but the regions analysed were in the contralateral breast or at a distance from the tumor. The peri-tumoral stroma region remains unexamined by IVIM.

McLaughlin et al. examined the diffusion signal in the peri-tumoral stroma following taxane treatment with a monoexponential model and measurements at two b-values [6]. They found a weak correlation between change in tumor volume and change in monoexponential apparent diffusion coefficient (ADC) from pretreatment values. Furthermore, there was no significant difference between ADC in stroma near $(2-5 \mathrm{~mm})$ and far $(9-13 \mathrm{~mm})$ from the tumor. This is somehwat puzzling since microvessel density decreases with distance from the tumor [3] and the protein content and density of the extracellular matrix also vary. This may be because ADC lacks specificity to microstructural changes: a decrease in vascular pseudodiffusion can be compensated for by faster diffusion in the extravascular space.

The DCE-MRI signal enhancement variations in the peri-tumoral stroma and the low specificity of monoexponential diffusion measures to vascular effects suggest that a more complex diffusion model may be needed in the stroma. This study modelled diffusion MRI data in breast using IVIM to examine vascular perfusion and tissue diffusion effects in the tumor and the peri-tumoral stroma. Results were compared to a monoexponential ADC method using the Akaike Information Criterion (AIC).

\section{Methods}

\subsection{Patients and Data Acquisition}

Three pre-chemotherapy patients presenting with breast cancer were imaged on a 3 T Siemens Trio (Siemens Healthcare, Erlangen) with a 7-channel InVivo breast coil in compliance with Local Research Ethics Committee approval.

Diffusion data were acquired with a 2D fat-saturated Twice-Refocused Spin Echo sequence at 5 b-values $\left(50,100,200,400-450\right.$ and $\left.800 \mathrm{~s} / \mathrm{mm}^{2}\right)$, with at least one unweighted image. Sequence parameters were: effective repetition time $\mathrm{TR}_{\text {eff }}=65 \mathrm{~ms}$, echo time $\mathrm{TE}=159 \mathrm{~ms}, 5$ averages, in-plane resolution $1.77 \mathrm{x}$ $1.77 \mathrm{~mm}^{2}$, slice thickness $4 \mathrm{~mm}$, field of view $12.7 \times 34.0 \mathrm{~cm}^{2}$ and $24-34$ slices.

DCE-MRI data were acquired with a 3D fat-saturated Spoiled Gradient Recalled sequence: $\mathrm{TR}=3.8 \mathrm{~ms}$, $\mathrm{TE}=1.38 \mathrm{~ms}, 1$ average, resolution $1.08 \times 1.08$ x $0.90 \mathrm{~mm}^{3}$, field of view 35.6 x 38.0 x $14.4 \mathrm{~cm}^{3}$. Patients received $0.1 \mathrm{mmol} / \mathrm{kg}$ body weight Gadoteric acid (Dotarem, Guerbet). Temporal resolution was $49 \mathrm{~s}$, 
but only the subtraction of the baseline image from the two-minute post-contrast image was used to define the tumor region.

\subsection{Registration, Region Selection and Noise Correction}

Motion was corrected by non-rigid registration of the unweighted images using NiftyReg (University College London, UK), a free-form registration implemented using cubic B-splines [7]. The transformation was then applied to the corresponding diffusion-weighted images in each direction.

Tumors were selected using a region-growing algorithm (MITK segmentation) on the DCE-MRI subtraction images and verified by a radiologist. These were transformed to the nearest diffusion-weighted slice and regions of interest (ROIs) at varying distances from the tumor (0-4, 4-8, 8-12 and 12-16 mm; see Figure 1) were generated. Each region was adjusted to exclude non-fibroglandular voxels based on a manual breast contour and decrease in signal of less than $30 \%$ from the 50 to $800 \mathrm{~s} / \mathrm{mm}^{2}$ images. Signal from voxels in each ROI was averaged to obtain a mean. Non-Gaussian noise was corrected by subtracting a factor dependent on the local noise [8]. All three diffusion-weighted directions were averaged to obtain signal for fitting and all unweighted image values were combined to get a single signal value for $b=0$ to use for fitting.

\subsection{Data Fitting}

Data were fitted to two different models: a monoexponential model

$$
S=S_{0} \exp (-b \cdot A D C),
$$

where $S_{0}$ is the signal in the absence of diffusion weighting and $A D C$ is the apparent diffusion coefficient; and the biexponential IVIM model

$$
S=S_{0}\left[f_{p} \exp \left(-b \cdot D_{p}\right)+\left(1-f_{p}\right) \exp \left(-b \cdot D_{t}\right)\right],
$$

where $S_{0}$ is as above, $f_{p}$ is the fraction of signal decay from pseudodiffusion, $D_{p}$ is the pseudodiffusion coefficient and $D_{t}$ is the tissue diffusion coefficient.

Weighted least squares fits were performed using Python and the scipy optimize minimize module with L-BFGS-B minimization. A lower bound of 0 was used for all parameters and upper bound of $5 \mathrm{x}$ the unweighted signal for $S_{0}$, no bound for $\mathrm{ADC}$ or $D_{t}$ and 1 for $f_{p}$. The data have low sensitivity to $D_{p}$, so it was fixed at $2 \times 10^{-2} \mathrm{~mm}^{2} / \mathrm{s}$ similar to previous work $[4,5]$.

The AIC indicates information lost by fitting while accounting for model complexity (lower values indicate less loss) and was calculated by

$$
A I C=\chi^{2}+2 k,
$$

where $\chi^{2}$ is the reduced chi-squared and $k$ is the number of fit parameters.

Parameter and AIC values were averaged across slices with visible tumor (n $=2,5$ and 1 for patients 1,2 and 3 respectively) from all patients. Voxel-by-voxel maps of the parameters were also generated. 
Statistical errors in the fitted parameters were calculated by adjusting one parameter at a time and re-fitting the remaining parameters until

$$
\chi^{2} \geq \chi_{0}^{2}\left[1+\frac{n_{p}}{N-n_{p}} F\left(n_{p}, N-n_{p}, 0.68\right)\right],
$$

where $\chi^{2}$ is the reduced chi-squared from the fit with one fixed parameter, $\chi_{0}^{2}$ is the reduced chi-squared from a fit where all parameters vary, $n_{p}$ is the number of parameters in the fit, $\mathrm{N}$ is the number of data points and $\mathrm{F}$ is the $\mathrm{F}$ distribution function, calculated here for a $68 \%$ confidence interval.

\section{Results}

Figure 1 shows sample DCE and diffusion-weighted $\left(b=200 \mathrm{~s} / \mathrm{mm}^{2}\right)$ images with the tumor outlined in cyan and the surrounding fibroglandular regions (0-4 mm, 4-8 $\mathrm{mm}, 8-12 \mathrm{~mm}$ and 12-16 $\mathrm{mm}$ ) in blue and purple. The mean SNR of the unweighted images was 69 and in the surrounding regions (increasing distance): 40, 42, 38 and 27. In the second patient, regions beyond $0 \mathrm{~mm}$ had low SNR and could not be analysed.
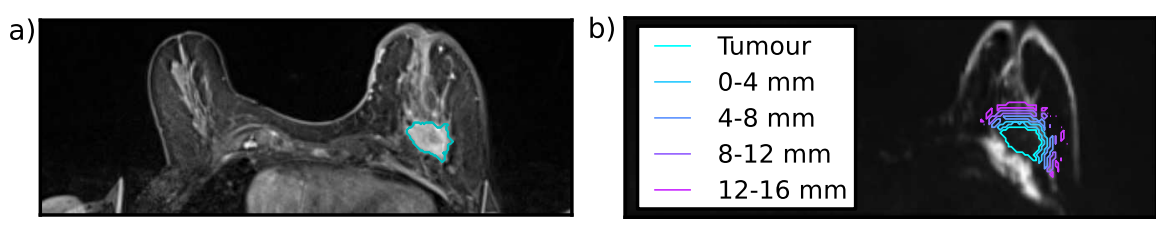

Fig. 1. (a) The post-contrast DCE and (b) diffusion-weighted ( $\left.b=200 \mathrm{~s} / \mathrm{mm}^{2}\right)$ images with the tumor (cyan) and surrounding ROIs of fibroglandular stroma outlined.

Figure $2 \mathrm{a}$ shows the fits of the models to the diffusion data for one slice of a tumor. Residuals are shown in 2b. Monoexponential fits are in solid blue (lines for the $68 \%$ confidence interval of the ADC parameter calculated by Eq. 4 are dotted) and biexponential fits are in red.

Figure 3 summarizes the parameters (mean +/- SD across the slices from all patients) from the monoexponential and biexponential fits as a function of distance from the tumor, as well as the AIC for each fit. Figure 4 shows maps of the fit parameters through one slice of the tumor volume. The $68 \%$ confidence intervals for the fit parameters in the tumor, given by Eq. 4 , were $4 \%$ for $S_{0}$ and $15 \%$ for $A D C$ for the monoexponential fit and $6 \%$ for $S_{0}, 25 \%$ for $D_{t}, 86 \%$ for the lower error of $f_{p}$ and $107 \%$ for the upper error of $f_{p}$ for the biexponential fit. Because the values of $f_{p}$ are relatively small, this translates to a parameter range of $0.02-0.21$. 

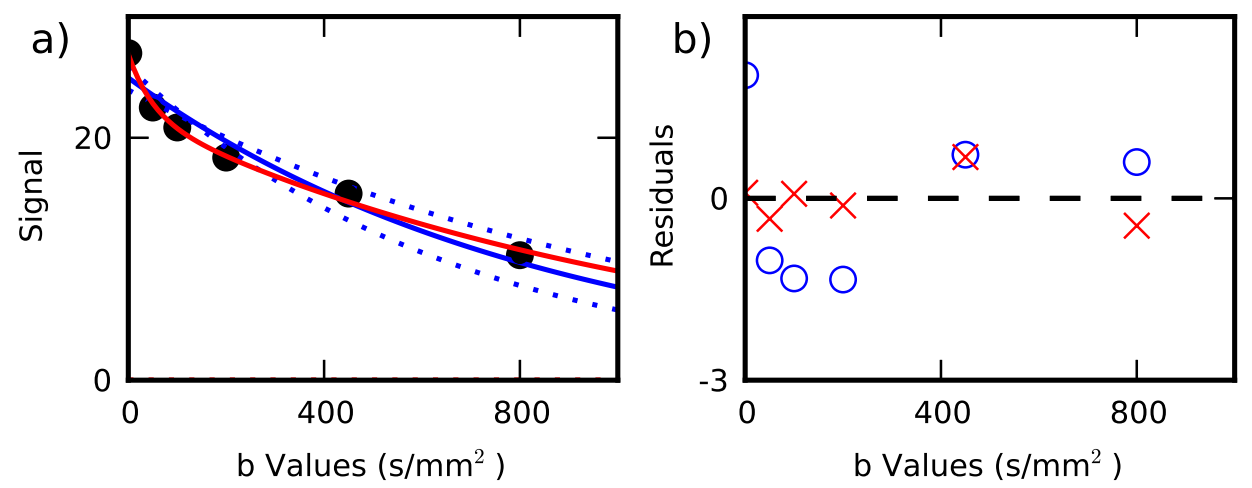

Fig. 2. Results of data fitting. (a) Signal as b value increases. Monoexponential fits to the data are in solid blue and biexponential fits are in red. The dotted line shows the fit using the ADC parameter error for the $68 \%$ confidence interval. (b) Residuals.
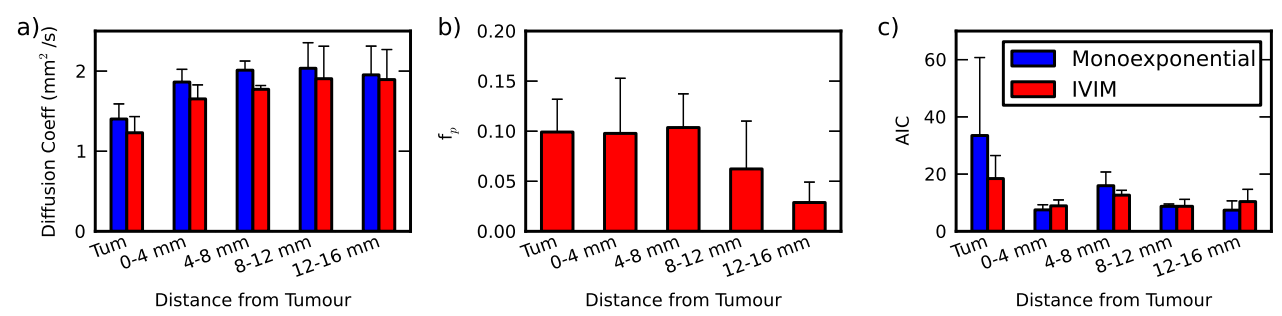

Fig. 3. Monoexponential (blue) and IVIM (red) fit parameters (a) ADC and $D_{t}$ in tumor and stromal regions and (b) fraction of pseudodiffusive signal (IVIM only). (c) AIC. Bar graphs mean +/- SD for all patients, all tumor-containing slices.

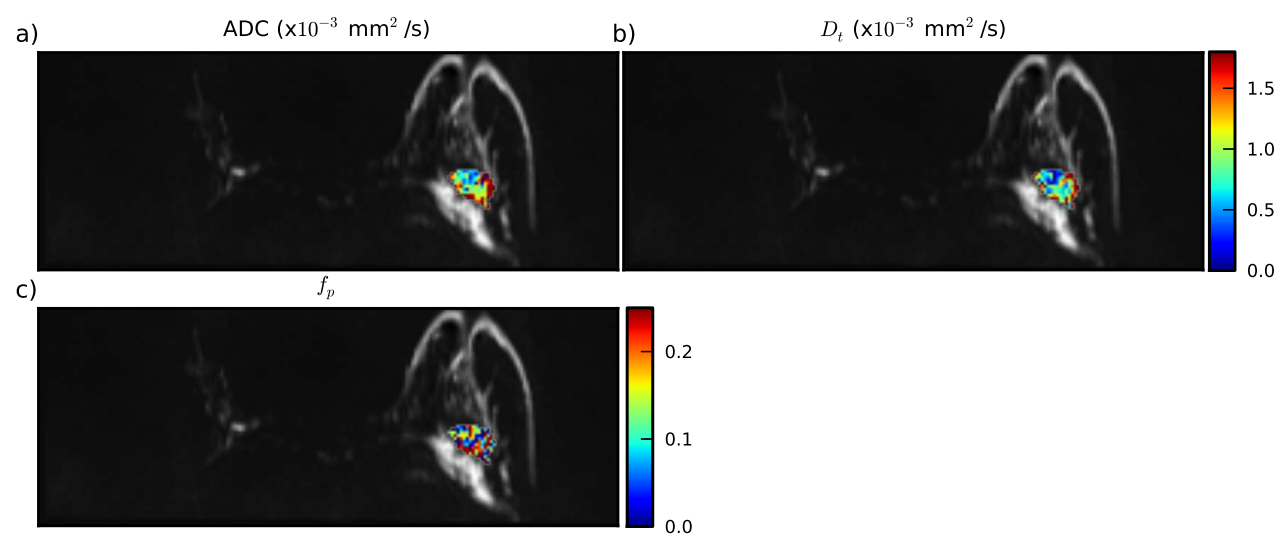

Fig. 4. Maps of fit parameters. (a) $A D C$ from the monoexponential fit, (b) $D_{t}$ from IVIM and (c) $f_{p}$ from IVIM 


\section{Discussion}

For peri-tumoral stroma within $8 \mathrm{~mm}$ of the tumor, IVIM provided a better fit to the diffusion data, shown by the lower and comparable AICs in this region, as well as the residuals (blue points in Figure $2 \mathrm{~b}$ ), which were consistently below zero for b-values of $100-200 \mathrm{~s} / \mathrm{mm}^{2}$ and then rose above zero. Residuals for the IVIM fit were more evenly distributed. IVIM did not add significant information in more distal stroma, however.

The values of the diffusion coeffecient $D_{t}=(1.4+/-0.2) \times 10^{-3} \mathrm{~mm}^{2} / \mathrm{s}$ and the pseudodiffusion fraction $f_{p}=0.10+/-0.03$ in the tumors themselves agreed with previous IVIM results: $D_{t}=(1.3+/-0.3) \times 10^{-3} \mathrm{~mm}^{2} / \mathrm{s}$ and $f_{p}=0.06+/-$ 0.03 in [4] and $D_{t}=(1.2+/-0.4) \times 10^{-3} \mathrm{~mm}^{2} / \mathrm{s}$ and $f_{p}=0.10+/-0.05$ in [5]. It should be noted that Bokacheva et al. excluded tumor edges, which tend to be more vascular, from their analysis, which may account for their slightly lower average. The parameter maps (Figure 4) demonstrated some heterogeneity of the parameters within the tumor. In addition, there may be differences in the patient populations.

In the stroma, $D_{t}$ was near that for free water and demonstrated an increasing trend further from the tumor, which could be due to less dense extracellular matrix or less cross-linking in this region. The monoexponential ADC showed little variation with distance from the tumor, in agreement with [6]. The pseudodiffusion fraction, $f_{p}$, decreased with distance from the tumor (Figure $3 \mathrm{~b}$ ), consistent with lower microvessel density [3] and previous DCE data [2]. These IVIM parameters suggest that the smaller contribution of vascular perfusion to the signal further from the tumor is compensated for by freer diffusion in the extravascular space, resulting in a similar calculated ADC value throughout the stroma when a two-point ADC method is used. Future work will attempt to validate the IVIM findings using histological measures of microvessel density.

The IVIM model assumes that the extravascular tissue can be represented by a single diffusion decay contribution. In the peri-tumoral stroma, there are very few cells relative to the tumor itself and low b-values are insensitive to cellular restriction, so this assumption is reasonable in this region. However, more complex models may be needed to describe the tumor, such as those incorporating restricted and hindered water diffusion by cells [9]. IVIM also assumes that water does not move between the vasculature and the tissue during measurement, which may affect parameter accuracy for leaky tumor vasculature.

This is the first study examining the diffusion signal in peri-tumoral stroma for non-monoexponential behavior. Although this preliminary work examined only a small number of patients, the results showed that a significant vascular contribution to the signal exists in the stroma close to the tumor. These differences could not be detected by a conventional monoexponential ADC model. In light of the correlations observed previously between DCE-MRI stromal enhancement and tumor recurrence, models of MR diffusion signal that account for vascular contributions deserve further study. 
Acknowledgements This work is supported by funding from EPSRC grant "MIMIC": EP/K020439/1 and EU FP7 Virtual Physiological Human grant: "VPH-PRISM" (FP7-ICT-2011-9, 601040).

\section{References}

1. Kim, M.Y., Cho, N., Koo, H.R., Yun, B.L., Bae, M.S., Chie, E.K., Moon, W.K.: Predicting local recurrence following breast-conserving treatment: parenchymal signal enhancement ratio (SER) around the tumor on preoperative MRI. Acta Radiol. 54, 731-738 (2013)

2. Hattangadi, J., Park, C., Rembert, J., Klifa, C., Hwang, J., Gibbs, J., Hylton, N.M.: Breast stromal enhancement on MRI is associated with response to neoadjuvant chemotherapy. Am. J. Roentgenol. 190, 1630-1636 (2008)

3. Nabavizadeh, N., Klifa, C., Newitt, D., Lu, Y., Chen, Y.-Y., Hsu, H., Fisher, C., Tokayasu, T., Olshen, A.B., Spellman, P., Gray, J.W. Hylton, N., Park, C. C.: Topographic enhancement mapping of the cancer-associated breast stroma using breast MRI. Integr. Biol. 3, 490-496 (2011)

4. Bokacheva, L., Kaplan, J.B., Giri, D.D., Patil, S., Gnanasigamani, M., Nyman, C.G., Deasy, J.O., Morris, E.A., Thakur, S.B.: Intravoxel incoherent motion diffusionweighted MRI at 3.0 $\mathrm{T}$ differentiates malignant breast lesions from benign lesions and breast parenchyma. J. Magn. Reson. Imaging. Early View (2013)

5. Sigmund, E.E., Cho, G.Y., Kim, S., Finn, M., Moccaldi, M., Jensen, J.H., Sodickson, D.K., Goldberg, J.D., Formenti, S., Moy, L.: Intravoxel incoherent motion imaging of tumor microenvironment in locally advanced breast cancer. Magn. Reson. Med. 65, 1437-1447 (2011)

6. Mclaughlin, R.L., Newitt, D.C., Wilmes, L.J., Jones, E.F., Wisner, D.J., Kornak, J., Proctor, E., Joe, B.N., Hylton, N.M.: High Resolution In Vivo Characterization of Apparent Difusion Coefficient at the Tumor-Stromal Boundary of Breast Carcinomas: A Pilot Study to Assess Treatment Response Using Proximity-Dependent Diffusion-Weighted Imaging. J. Magn. Reson. Imaging. Early View (2013)

7. Modat, M., Ridgway, G.R., Taylor, Z.A., Lehmann, M., Barnes, J., Fox, N.C., Hawkes, D.J., Ourselin,S.: Fast free-form deformation using graphics processing units. Comput. Methods Programs Biomed. 98, 278-284 (2010)

8. Henkelman, R.M.: Measurement of signal intensities in the presence of noise in MR images. Med. Phys. 12, 232-233 (1985)

9. Panagiotaki, E., Schneider, T., Siow, B., Hall, M.G., Lythgoe, M.F., Alexander, D.C.: Comparment models of the diffusion MR signal in brain white matter: a taxonomy and comparison. NeuroImage. 59, 2241-2254 (2012) 